\title{
The Performance of a Modified Glasgow Blatchford Score in Predicting Clinical Interventions in Patients with Acute Nonvariceal Upper Gastrointestinal Bleeding: A Vietnamese Prospective Multicenter Cohort Study
}

\author{
Duc Trong Quach* ${ }^{,}{ }^{\dagger}$, Ngoi Huu Dao ${ }^{\ddagger}$, Minh Cao Dinh${ }^{\S}$, Chung Huu Nguyen* ${ }^{, \prime}$, Linh Xuan Ho ${ }^{\dagger}$, Nha-Doan Thi Nguyen*,", \\ Quang Dinh Le ${ }^{*}{ }^{\dagger}$, Cong Minh Hong $\mathrm{Vo}^{\dagger}$, Sang Kim Le ${ }^{\prime \prime}$, and Toru Hiyama\# \\ *Department of Internal Medicine, University of Medicine and Pharmacy, Hochiminh City, ${ }^{\dagger}$ Department of Gastroenterology, Gia-Dinh People’s \\ Hospital, ${ }^{\ddagger}$ Department of Gastroenterology, An-Binh Hospital, ${ }^{s}$ Department of Gastroenterology, Dong-Nai General Hospital, "Department of \\ Gastroenterology, Trung-Vuong Emergency Center, "Department of Gastroenterology, Nguyen-Tri-Phuong Hospital, Ho Chi Minh, Vietnam, \\ and "Health Service Center, Hiroshima University, Higashihiroshima, Japan
}

Background/Aims: To compare the performance of a modified Glasgow Blatchford score (mGBS) to the Glasgow Blatchford score (GBS) and the pre-endoscopic Rockall score (RS) in predicting clinical interventions in Vietnamese patients with acute nonvariceal upper gastrointestinal bleeding (ANVUGIB). Methods: A prospective multicenter cohort study was conducted in five tertiary hospitals from May 2013 to February 2014. The mGBS, GBS, and pre-endoscopic RS scores were prospectively calculated for all patients. The accuracy of mGBS was compared with that of GBS and preendoscopic RS using area under the receiver operating characteristic curve (AUC). Clinical interventions were defined as blood transfusions, endoscopic or radiological intervention, or surgery. Results: There were 395 patients including 128 (32.4\%) needing endoscopic treatment, 117 (29.6\%) requiring blood transfusion and two $(0.5 \%)$ needing surgery. In predicting the need for clinical intervention, the mGBS (AUC, 0.707 ) performed as well as the GBS (AUC, 0.708; $p=0.87$ ) and outperformed the pre-endoscopic RS (AUC, 0.594; $p<0.001)$. However, none of these scores effectively excluded the need for endoscopic intervention at a threshold of 0 . Conclusions: mGBS performed as well as GBS and better than pre-endoscopic RS for predicting clinical interventions in Vietnamese patients with ANVUGIB. (Gut Liver 2016;10:375381)

Key Words: Gastrointestinal hemorrhage; Prediction score; Prognosis; Vietnamese

\section{INTRODUCTION}

Acute nonvariceal upper gastrointestinal bleeding (ANVUGIB) is a common emergency problem in clinical practice. Stratifying patients based on the needs for clinical interventions (i.e., blood transfusion, endoscopic or radiological intervention, or surgery) is among the key issues to optimize the management strategy and, therefore, have been recently intensively studied. The Glasgow Blatchford score (GBS) and the Rockall score (RS) have been recommended in several guidelines as the useful tools to predict the needs for clinical interventions in patients with ANVUGIB. ${ }^{1,2}$ In areas with limited endoscopic resources, accurately predicting the need for endoscopic intervention is especially useful for patient allocation. The Asia-Pacific consensus for ANVUGIB management recommends to use GBS for this purspose. However, this recommendation was based on only one singlecenter cohort study in Hong Kong which showed that there were no endoscopic intervention needed at the threshold 0 of Blachford score. ${ }^{4}$ Therefore, more studies in other Asian populations are needed.

In clinical practice, the routine use of GBS may be complicated by the presence of subjective parameters that are open to interpretation. Recently, a study in the United States reported that a modified GBS (mGBS) which eliminated the subjective criteria of GBS (i.e., syncope, melena and the prior history of co-existent liver disease or heart failure) was comparable with GBS in predicting the needs for clinical interventions. ${ }^{5}$ However, this is a single-center study with only $12.5 \%$ of patients in the study were of Asian descent. In addition, the main objective of the study was to evaluate the role of the modified score in pre-

Correspondence to: Duc Trong Quach

Department of Internal Medicine, University of Medicine and Pharmacy, Hochiminh City, 215 Hong Bang, District 5, Ho Chi Minh 70000, Vietnam

Tel: +84-8-38554269, Fax: +84-8-39506126, E-mail: drquachtd@ump.edu.vn

Received on June 8, 2015. Revised on July 4, 2015. Accepted on July 15, 2015. Published online November 27, 2015

pISSN 1976-2283 eISSN 2005-1212 http://dx.doi.org/10.5009/gnl15254

(c) This is an Open Access article distributed under the terms of the Creative Commons Attribution Non-Commercial License (http://creativecommons.org/licenses/by-nc/4.0) which permits unrestricted non-commercial use, distribution, and reproduction in any medium, provided the original work is properly cited. 
dicting clinical interventions and not in predicting endoscopic intervention. We, therefore, conducted this prospective multicenter study to evaluate the role of mGBS, in comparison with GBS and the pre-endoscopic RS, in predicting the needs for clinical and endoscopic interventions in Vietnamese population.

\section{MATERIALS AND METHODS}

\section{Setting and study design}

Multicenter prospective cohort study conducted at five tertiary medical centers in Vietnam (Gia-Dinh People's Hospital, An-Binh Hospital, Dong-Nai General Hospital, Trung-Vuong Emergency Center, and Nguyen-Tri-Phuong Hospital). The study was approved by the ethic committee of each center.

\section{Study population}

All patients $>18$ years of age presenting to the emergency room with overt symptoms of upper gastrointestinal bleeding (UGIB) (i.e., hematemesis or coffee-ground emesis, melena, hematochezia with positive nasogastric lavage) from nonvariceal source were eligible for inclusion. Endoscopy was offered to all patients. Exclusion criteria included: patients who have been resuscitated and referred from another hospital, recurrent UGIB within the study period, patients who developed UGIB while admitted to the hospital for other reasons, or patients who could not be performed upper gastrointestinal endoscopy within 24 hours after admission. All patients presenting to the emergency room with signs and symptoms of UGIB were monitored for hospital outcomes while admitted.

\section{Data collection}

Data collected at admission included demographic information, vital signs, physical exam findings, laboratory values, medical comorbidities, endoscopic findings, and clinical interventions (i.e., blood transfusion, endoscopic or radiological intervention, or surgery). In addition, rebleeding and in-hospital mortality were recorded.

\section{Calculation of scoring systems}

The mGBS, GBS, and pre-endoscopic RS were calculated on each patient on admission. The mGBS incorporates only the quantitative parameters of the GBS: pulse, systolic blood pressure, blood urea nitrogen, and hemoglobin (Hb) (Table 1).

In this study, we apply the following criteria for the subjective parameters of the GBS and pre-endoscopic RS: (1) cardiac failure: clinical signs of decompensated congestive heart failure, diastolic dysfunction on echocardiogram or ejection fraction of $<45 \%$; (2) hepatic disease or failure: clinical signs of portal hypertension and hepatic failure and/or imaging characteristic of cirrhosis; (3) ischemic heart disease: active angina with electrocardiogram showing signs of ischemia; (4) chronic kidney disease: glomerular filtration rate of $<60 \mathrm{~mL} / \mathrm{min} / 1.73 \mathrm{~m}^{2}$; or (5) melena: presence left to the judgment of the evaluating physician.

\section{Treatment}

This is an observation study. It is the standard at all studying hospitals to start intravenous proton pump inhibitor such as esomeprazole, omeprazole, or pantoprazole in the emergency room for patients presenting with UGIB. The choice of intermittent or continuous infusion was left to the discretion of the physicians in charge. Blood transfusion was generally decided in following situations: (1) patients presented with signs of significantly hemodynamic instability (i.e., pulse $\geq 120$ beats per minute or systolic blood pressure $<90 \mathrm{~mm} \mathrm{Hg}$ ); (2) patients with $\mathrm{Hb}<7 \mathrm{~g} / \mathrm{dL}(\mathrm{Hb}<8 \mathrm{~g} / \mathrm{dL}$ if patients were $\geq 65$ years old, with coexistent cardiac ischemia or chronic obstructive pulmonary diseases). Upper gastrointestinal endoscopy were performed within 24 hours after patients were admitted to the emergency room. The indications for endoscopic interventions in this study were peptic ulcers with Forrest Ia, Ib, IIa, and IIb classification. For other nonvariceal bleeding sources, endoscopic interventions

Table 1. Scoring Systems

\begin{tabular}{|c|c|c|}
\hline Scoring system & Clinical factor & Score \\
\hline Modified Glasgow & Pulse, beats per minutes & \\
\hline \multirow[t]{17}{*}{ Blatchford score } & $\geq 100$ & 1 \\
\hline & Systolic blood pressure, mm Hg & \\
\hline & $100-109$ & 1 \\
\hline & $90-99$ & 2 \\
\hline & $<90$ & 3 \\
\hline & Blood urea nitrogen, mg/dL & \\
\hline & 19 and $<22.4$ & 2 \\
\hline & $\geq 22.4$ and $<28$ & 3 \\
\hline & $\geq 28$ and $<70$ & 4 \\
\hline & $\geq 70$ & 6 \\
\hline & Hemoglobin (male), g/dL & \\
\hline & $\geq 12.0$ and $<13$ & 1 \\
\hline & $\geq 10.0$ and $<12$ & 3 \\
\hline & $<10$ & 6 \\
\hline & Hemoglobin (female), g/dL & \\
\hline & $\geq 10$ and $<12$ & 1 \\
\hline & $<10$ & 6 \\
\hline Full Glasgow Blatchford & Chronic disease/major comorbidities & \\
\hline score (includes these & Hepatic disease & 2 \\
\hline \multirow[t]{5}{*}{ additional factors) } & Cardiac failure & 2 \\
\hline & Melena & \\
\hline & Present & 1 \\
\hline & Syncope & \\
\hline & Present & 2 \\
\hline
\end{tabular}




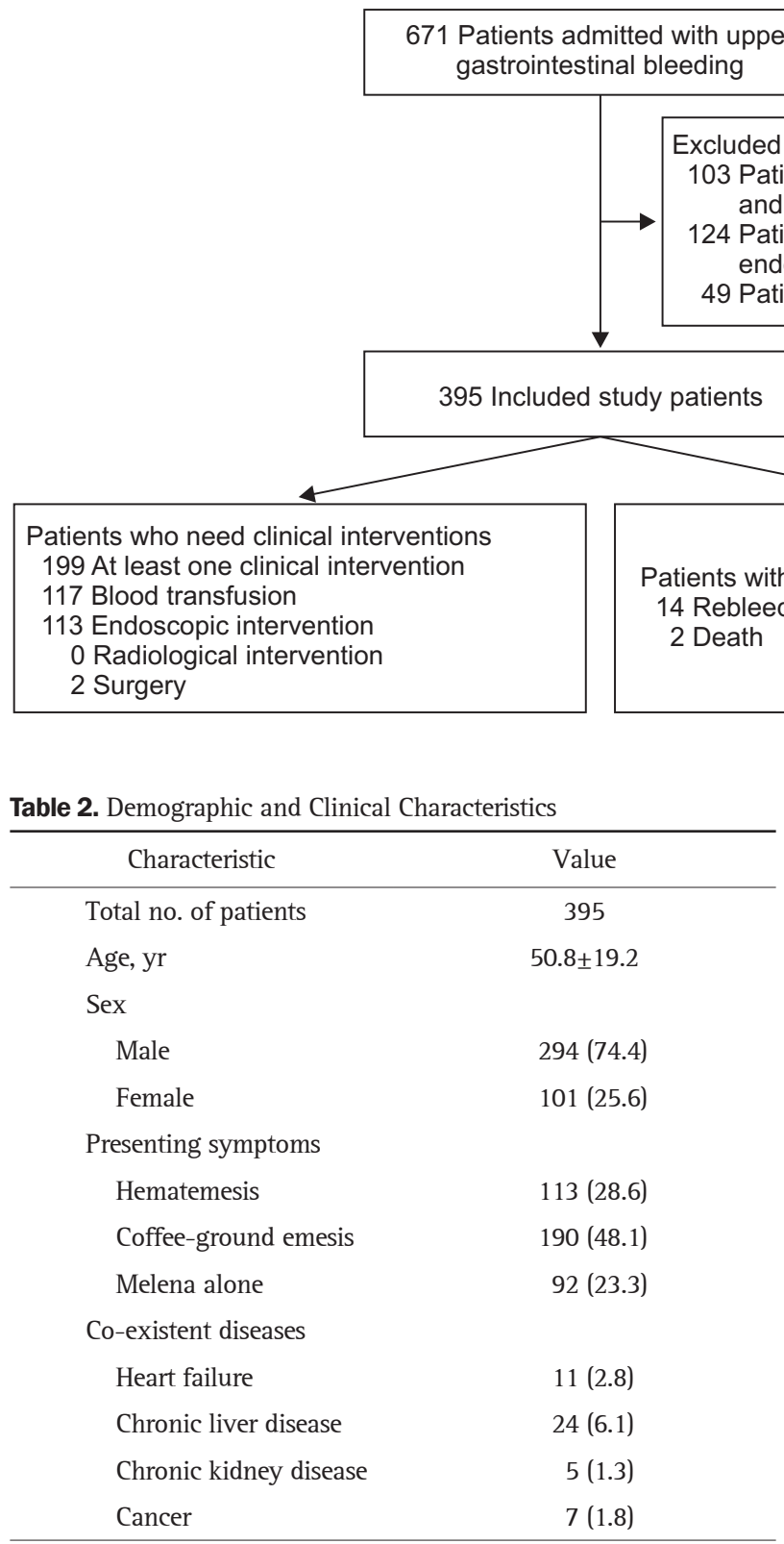

Data are presented as mean \pm SD or number $(\%)$.

were decided by the physician in charge.

\section{Outcomes}

The primary outcome was the need for clinical interventions, which was a composite of blood transfusion, endoscopic, radiological intervention, and surgery.

\section{Statistical analysis}

The mGBS was compared with the GBS, and pre-endoscopic RS for the above-mentioned outcome. Accuracy of each scoring system was measured using the area under the receiver operating characteristic curve (AUC). The chi-square test was used to compare the difference between AUC curves according to the method described by DeLong et al. ${ }^{6}$ Data analysis was per-
Table 3. Endoscopic Findings

\begin{tabular}{lc}
\hline Bleeding causes & No. of patients (\%) \\
\hline Gastric ulcer & $149(37.7)$ \\
Duodenal ulcer & $142(35.9)$ \\
Mallory Weiss tear & $38(9.6)$ \\
Erosive gastritis & $21(5.3)$ \\
Gastric cancer & $7(1.8)$ \\
Esophageal ulcer & $7(1.8)$ \\
Dieulafoy lesion & $3(0.8)$ \\
Unidentified & $28(7.1)$ \\
\hline
\end{tabular}

formed using SAS 9.2 (SAS Inc., Cary, NC, USA). All reported pvalues were two-sided with $\alpha<0.05$ threshold for significance.

\section{RESULTS}

From May 2013 to February 2014, consecutive patients with UGIB admitted to the five study centers were recruited. A total of 395 patients who fulfilled the inclusion criteria were included in the study analysis (Fig. 1). For the outcomes, 199 patients (54.4\%) had at least one clinical intervention which included 117 (29.6\%) who required blood transfusion, 133 (33.7\%) endoscopic intervention, no (0\%) radiological intervention and two $(0.5 \%)$ surgical intervention. Regarding the types of endoscopic interventions, 103 (26.1\%) were with epinephrine injection alone and 30 (7.6\%) were with combination therapy (epinephrine injection plus clipping/thermotherapy). There were 14 (3.5\%) inhospital rebleeding and two (0.5\%) in-hospital death.

The mGBS, GBS and pre-endoscopic RS were calculated on all of these patients. Table 2 depicts the baseline demographics and clinical characteristics of patients in the study. The mean age of recruited patients was 50.8 \pm 19.2 , with 101 women (25.6\%). 
Table 3 lists the bleeding lesions detected by endoscopy.

\section{Accuracy of scoring systems}

1) Accuracy of scoring systems in predicting clinical interventions

The mGBS (AUC, 0.708; 95\% confidence interval [CI], 0.660 to 0.752 ) performed as well as the GBS (AUC, 0.707; 95\% CI, 0.661 to $0.753 ; \mathrm{p}=0.87$ ), and outperformed the pre-endoscopic RS (AUC, $0.594 ; 95 \%$ CI, 0.544 to $0.643 ; \mathrm{p}=0.0001$ ) for predicting clinical interventions.

\section{2) Accuracy of scoring systems in predicting endoscopic} interventions

The mGBS (AUC, $0.608 ; 95 \% \mathrm{CI}, 0.562$ to 0.660 ) performed as well as the GBS (AUC, 0.612; 95\% CI, 0.558 to 0.656; $\mathrm{p}=0.55$ ) and outperformed the pre-endoscopic RS (AUC, 0.539; 95\% CI, 0.489 to $0.589 ; \mathrm{p}=0.02$ ) for predicting endoscopic interventions (Table 4).

The presence of endoscopic lesions with high-risk stigmata according to scoring systems is depicted in Fig. 2. 42.8\% patients (6/14) with GBS score $=0 ; 32.2 \%$ patients (10/31) with $\mathrm{mGBS}=0$ and $30 \%$ patients $(36 / 120)$ with pre-endoscopic $\mathrm{RS}=0$

\section{A}

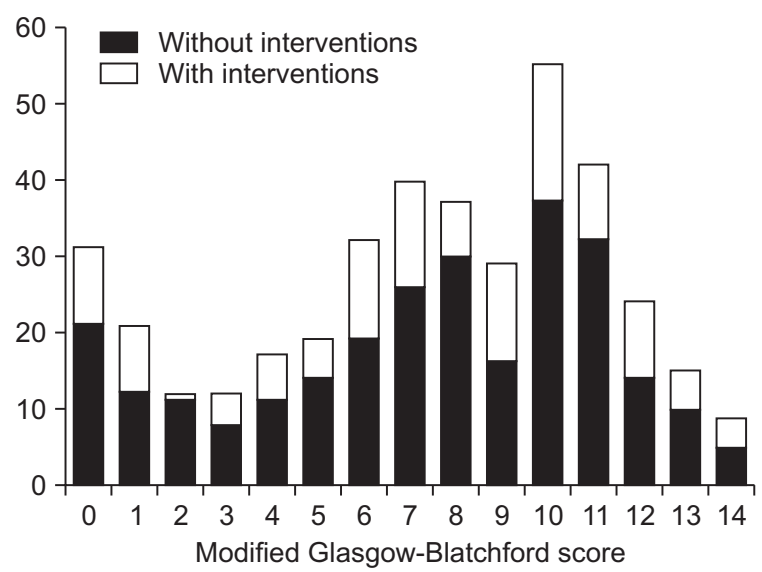

C

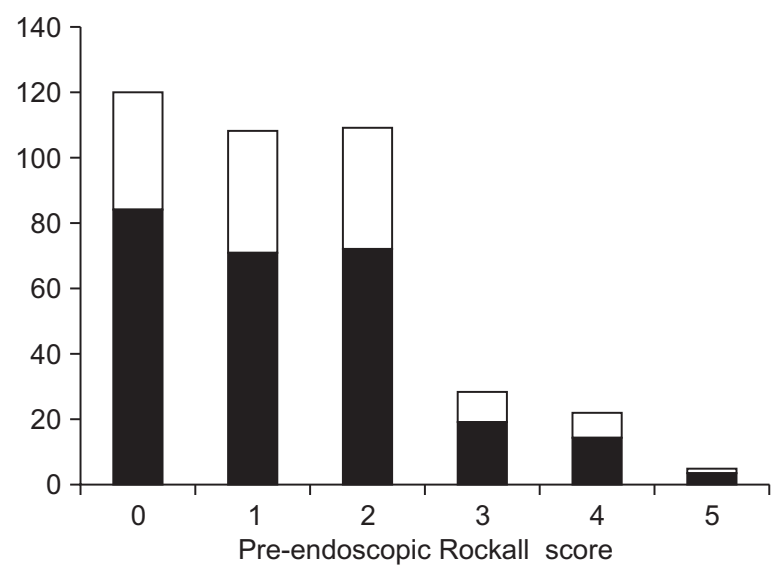

had high-risk endoscopic stigmata which required endoscopic interventions. Therefore, our study showed that no scores can effectively help to rule out the possibility of endoscopic interventions at the threshold of 0 point.

Table 4. Accuracy of Scoring Systems for Predicting Clinical and Endoscopic Interventions

\begin{tabular}{lccc}
\hline & AUC & $95 \% \mathrm{CI}$ & $\begin{array}{c}\text { Comparison } \\
\text { to mGBS } \\
\text { (p-value) }\end{array}$ \\
\hline $\begin{array}{l}\text { Clinical interventions } \\
\text { mGBS }\end{array}$ & 0.708 & $0.660-0.752$ & - \\
GBS & 0.707 & $0.661-0.753$ & 0.87 \\
$\quad$ Pre-endoscopic RS & 0.594 & $0.544-0.643$ & $<0.01$ \\
Endoscopic interventions & & & \\
$\quad$ mGBS & 0.608 & $0.562-0.660$ & - \\
$\quad$ GBS & 0.612 & $0.558-0.656$ & 0.55 \\
Pre-endoscopic RS & 0.539 & $0.489-0.589$ & 0.02 \\
\hline
\end{tabular}

AUC, area under the receiver operating characteristic curve; CI, confidence interval; mGBS, modified Glasgow Blatchford score; GBS, Glasgow Blatchford score; RS, Rockall score.

B

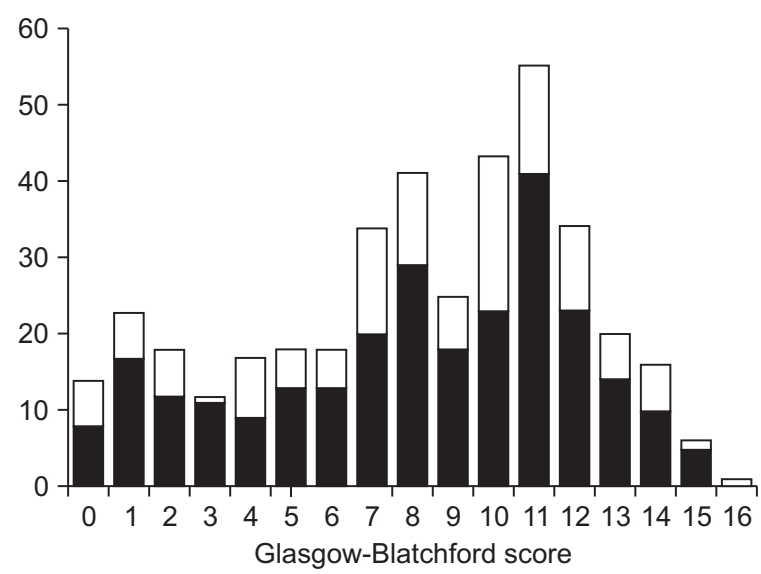

Fig. 2. Distribution of the modified Glasgow-Blatchford score (A), Glasgow Blatchford score (B), and pre-endoscopic Rockall score (C) stratified according to the need for endoscopic intervention. 


\section{DISCUSSION}

UGIB is a common clinical problem and accounts for 25 to 35 hospitalizations per 100,000 person-years. ${ }^{7}$ Accurately predicting the outcome of UGIB will help to optimize clinical management. Current guidelines recommend that risk assessment should be performed to stratify patients into high and low risk categories because it may assist in initial decisions such as timing of endoscopy, patient allocation, time of discharge and level of care. ${ }^{1,2}$ The popular instruments used to assess risk include GBS and pre-endoscopic RS which use only clinical and laboratory data before endoscopy. Both of these two scores include pulse and systolic pressure as parameters. As our study was conducted at tertiary hospitals, we have excluded all patients presenting with UGIB who have been resuscitated and transferred from other hospitals in order to accurately calculate these scores at admission time. Our study showed that GBS outperformed pre-endoscopic RS in predicting clinical interventions (AUC, 0.707 vs 0.594; $\mathrm{p}=0.0001$ ). Previous studies in other populations have also shown similar results. ${ }^{8-10}$ Chen et al. ${ }^{8}$ retrospectively evaluated 354 patients in Taiwan presenting with UGIB. This study showed that GBS identified patients at high risk for clinical interventions (blood transfusion, endoscopic or surgical intervention) better compared with the pre-endoscopic RS (92.1\% vs $81.6 \%, \mathrm{p}<0.0001)$. Stanley et al. ${ }^{9}$ prospectively studied 1,555 consecutive patients in four U.K. hospitals. This study showed that GBS outperformed the pre-endoscopic RS (AUC, 0.944 vs $0.756 ; \mathrm{p}<0.00005$ ) in predicting the need for transfusion and endoscopic or surgical intervention (AUC, 0.858 vs 0.705; $\mathrm{p}<0.00005)$. More recently, Chandra et al. ${ }^{10}$ reported a retrospective study on 171 consecutive patients presented with UGIB at an academic emergency department in the United States. This study also showed that GBS outperformed pre-en- doscopy RS (AUC, 0.79 vs $0.62 ; p=0.0001$ ) but the specificity of both scores was suboptimal at all potential decision thresholds so that it was insufficient to recommend using of either score in clinical practice.

The difficulty to apply GBS in clinical practice is that it incorporates some subjective parameters, such as cardiac failure and hepatic disease. The severity and the clear-cut definition of how to evaluate these parameters were not well-defined in the original score, which may lead to different calculation in the same patient when being scored by different physicians. Cheng et al., therefore, have suggested the use of mGBS that only incorporates quantitative parameters (vital signs and laboratory studies). The mGBS (AUC, 0.87) has shown to perform as well as the GBS (AUC, 0.88) p=0.44, and outperform the pre-endoscopic RS (AUC, 0.68) $\mathrm{p}<0.0001$ in patients with ANVUGIB. This modified score could be even more potentially useful in areas with limited medical resources where the presence of cardiac failure and hepatic disease in patients admitted with acute UGIB has to be judged based on clinical findings only. The results of our study showed that the mGBS performed as well as GBS and better than the pre-endoscopic RS in predicting clinical interventions in Vietnamese population.

According to current guidelines on the management of patients with UGIB, patients suspected for UGIB admitted to the hospital should be performed early endoscopy (i.e., within 24 hours after presentation). ${ }^{1-3}$ This is because observational studies do suggest a benefit of endoscopy within 24 hours after admission in terms of decreased length of stay, ${ }^{11,12}$ and surgical intervention. ${ }^{12}$ Meta-analysis of trials of endoscopic therapy vs no endoscopic therapy for patients with high-risk endoscopic lesions (i.e., actively bleeding ulcer or nonbleeding visible vessel in an ulcer) also shows a significant decrease in further bleeding, the need for urgent intervention and surgery. ${ }^{13}$ As
A

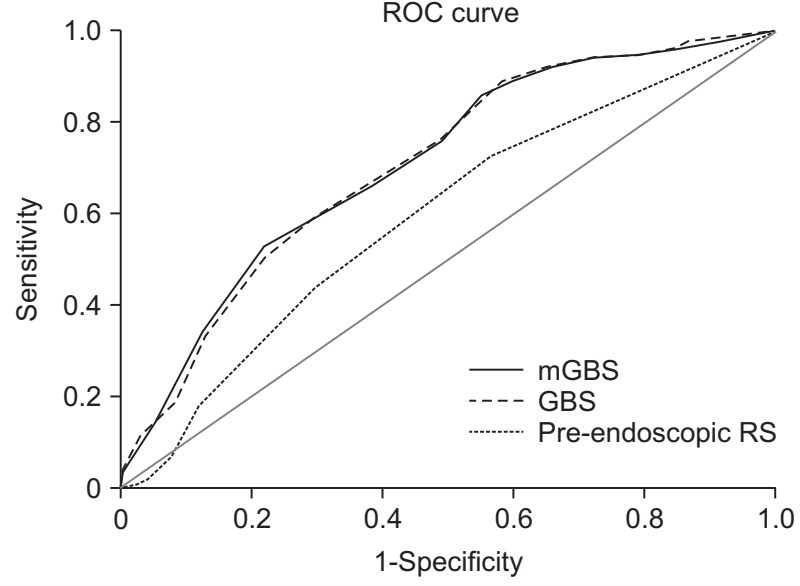

B

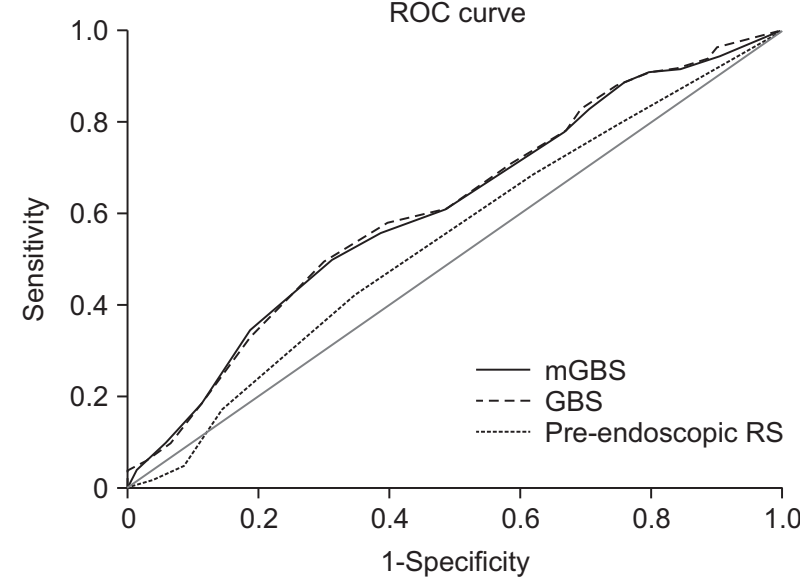

Fig. 3. Comparison of the modified Glasgow Blatchford score (mGBS), Glasgow Blatchford score (GBS), and pre-endoscopic Rockall score (RS). Area under the receiver operating characteristic curve of each scoring system to predict clinical intervention (blood transfusion, endoscopic or radiological intervention or surgery) (A) and endoscopic intervention alone (B).

ROC, receiver operating characteristic. 
endoscopic interventions may be not available in some general clinics, especially in developing countries, accurate predicting of high-risk endoscopic stigmata is very helpful to decide whether patients with UGIB need to be transferred to another hospital with endoscopic intervention unit. Regarding to this issue, Pang et al. ${ }^{4}$ conducted a single-center prospective cohort in Hong Kong which showed that GBS was more useful than pre-endoscopic RS in predicting low-risk patients. In this study, no patients required endoscopic interventions at the GBS score of 0 . In our study, the mGBS performed as well as the GBS and outperformed the pre-endoscopic RS. However, none of these scores could safely rule out the possibility of have high-risk endoscopic lesions (Fig. 2). Fig. 3 also showed that the specificity of mGBS and GBS to predict clinical and endoscopic interventions was suboptimal at all potential decision thresholds. Therefore, applying these scores alone may suggest but cannot accurately predict the need for clinical and endoscopic interventions in Vietnamese population. Recently, Farooq et al. ${ }^{14}$ reported a study on 195 patients with UGIB in the United States, which showed that the accuracy of the GBS and pre-endoscopic RS might not be as good as clinical decision making by emergency physician. However, the weak point of this study is its retrospective and single-center design. In addition, it was conducted in one tertiary medical center where the physicians might be generally more experienced than the ones in charge of patients with UGIB in the real scenario. De Groot et al. ${ }^{15}$ reported the added value of senior gastroenterologists' feeling to prediction scores in patients with a suspected UGIB. This prospective study which included 974 patients with UGIB was conducted at eight hospitals in the Netherlands. The result showed that GBS was better than gastroenterologists' feeling, but combining both GBS and the gastroenterologists' feeling yielded the highest predictive power for the need of clinical interventions. The prediction of endoscopic intervention in this study, however, was not separately analyzed. This data suggests that using mGBS and taking physician's experience into consideration may lead to more accurate prediction of clinical and endoscopic interventions. Future studies are awaited to clarify this issue.

Our study has several strengths. Firstly, this is the first prospective multicenter study showing that a mGBS performs as well as GBS and better than pre-endoscopic RS in the prediction of clinical and endoscopic interventions in Asia. Secondly, we have applied a strictly exclusion criteria which include patients who has been resuscitated and referred from another hospital and who could not be performed upper gastrointestinal endoscopy within 24 hours after admission. The calculations of prediction scores, therefore, would be accurate. On the other hand, the limitation of this study is that we have applied arbitrary criteria for subjective parameters of GBS which may be different from other studies. In addition, all patients recruited in our study were of Vietnamese descent. Therefore, the performance of the mGBS should be validated in other populations.
In summary, our study showed that the mGBS that only contains the four quantitative components of the original GBS performed as well as the GBS and outperformed the pre-endoscopic RS in Vietnamese patients presenting with ANVUGIB. We believe that this mGBS will be easier to use in clinical practice.

\section{CONFLICTS OF INTEREST}

No potential conflict of interest relevant to this article was reported.

\section{ACKNOWLEDGEMENTS}

We thank Drs Phong Ha and Hung Le for their kindly advice to prepare the manuscript. The publication of this paper was supported by AstraZeneca Vietnam.

Author contributions: Concept/design: DT Quach, T Hiyama; Conduct of study: DT Quach, NH Dao, MC Dinh, CH Nguyen, DNT Nguyen, QD Le, CMH Vo, SK Le; Data analysis/interpretation: DT Quach, QD Le; Drafting article: DT Quach, T Hiyama; Critical revision of article: DT Quach, T Hiyama; Approval of article: all authors.

\section{REFERENCES}

1. Barkun AN, Bardou M, Kuipers EJ, et al. International consensus recommendations on the management of patients with nonvariceal upper gastrointestinal bleeding. Ann Intern Med 2010;152:101113.

2. Laine L, Jensen DM. Management of patients with ulcer bleeding. Am J Gastroenterol 2012;107:345-360.

3. Sung JJ, Chan FK, Chen M, et al. Asia-Pacific Working Group consensus on non-variceal upper gastrointestinal bleeding. Gut 2011;60:1170-1177.

4. Pang SH, Ching JY, Lau JY, Sung JJ, Graham DY, Chan FK. Comparing the Blatchford and pre-endoscopic Rockall score in predicting the need for endoscopic therapy in patients with upper GI hemorrhage. Gastrointest Endosc 2010;71:1134-1140.

5. Cheng DW, Lu YW, Teller T, Sekhon HK, Wu BU. A modified Glasgow Blatchford Score improves risk stratification in upper gastrointestinal bleed: a prospective comparison of scoring systems. Aliment Pharmacol Ther 2012;36:782-789.

6. DeLong ER, DeLong DM, Clarke-Pearson DL. Comparing the areas under two or more correlated receiver operating characteristic curves: a nonparametric approach. Biometrics 1988;44:837-845

7. Lanas A, García-Rodríguez LA, Polo-Tomás M, et al. The changing face of hospitalisation due to gastrointestinal bleeding and perforation. Aliment Pharmacol Ther 2011;33:585-591.

8. Chen IC, Hung MS, Chiu TF, Chen JC, Hsiao CT. Risk scoring systems to predict need for clinical intervention for patients with nonvariceal upper gastrointestinal tract bleeding. Am J Emerg Med 2007;25:774-779. 
9. Stanley AJ, Dalton HR, Blatchford O, et al. Multicentre comparison of the Glasgow Blatchford and Rockall Scores in the prediction of clinical end-points after upper gastrointestinal haemorrhage. Aliment Pharmacol Ther 2011;34:470-475.

10. Chandra S, Hess EP, Agarwal D, et al. External validation of the Glasgow-Blatchford Bleeding Score and the Rockall Score in the US setting. Am J Emerg Med 2012;30:673-679.

11. Cooper GS, Chak A, Connors AF Jr, Harper DL, Rosenthal GE. The effectiveness of early endoscopy for upper gastrointestinal hemorrhage: a community-based analysis. Med Care 1998;36:462-474.

12. Cooper GS, Chak A, Way LE, Hammar PJ, Harper DL, Rosenthal GE. Early endoscopy in upper gastrointestinal hemorrhage: as- sociations with recurrent bleeding, surgery, and length of hospital stay. Gastrointest Endosc 1999;49:145-152.

13. Laine L, McQuaid KR. Endoscopic therapy for bleeding ulcers: an evidence-based approach based on meta-analyses of randomized controlled trials. Clin Gastroenterol Hepatol 2009;7:33-47.

14. Farooq FT, Lee MH, Das A, Dixit R, Wong RC. Clinical triage decision vs risk scores in predicting the need for endotherapy in upper gastrointestinal bleeding. Am J Emerg Med 2012;30:129-134.

15. de Groot N, van Oijen M, Kessels K, et al. Prediction scores or gastroenterologists' Gut Feeling for triaging patients that present with acute upper gastrointestinal bleeding. United European Gastroenterol J 2014;2:197-205. 